\title{
L'espace métagénétique : exogenèse, intertextualité, écritures de soi
}

Véronique Montémont et Françoise Simonet-Tenant

\section{(2) OpenEdition}

1 Journals

Édition électronique

URL : https://journals.openedition.org/genesis/5667

DOI : 10.4000/genesis.5667

ISSN : 2268-1590

Éditeur :

Presses universitaires de Paris Sorbonne (PUPS), Société internationale de génétique artistique littéraire et scientifique (SIGALES)

Édition imprimée

Date de publication : 15 décembre 2020

Pagination : 51-61

ISBN : 979-10-231-0704-3

ISSN : 1167-5101

\section{Référence électronique}

Véronique Montémont et Françoise Simonet-Tenant, « L'espace métagénétique : exogenèse,

intertextualité, écritures de soi », Genesis [En ligne], 51 | 2020, mis en ligne le 20 décembre 2021, consulté le 07 février 2022. URL : http://journals.openedition.org/genesis/5667 ; DOI : https://doi.org/ 10.4000/genesis.5667 


\title{
L'espace métagénétique : exogenèse, intertextualité, écritures de soi
}

\author{
Véronique Montémont et Françoise Simonet-Tenant
}

$\mathrm{L}$ es écritures de soi, bien qu'il soit désormais admis qu'elles relèvent pleinement de la littérature, demeurent des objets particuliers au regard de l'étude génétique. Leurs enjeux spécifiques ont été explorés par Philippe Lejeune, en particulier dans Les Brouillons de soi (1998) et Autogenèses (2013), deux ouvrages qui mettent en avant le caractère doublement déterminant d'une lecture génétique, qui peut être appliquée à l'autobiographie stricto sensu, mais aussi aux journaux, aux correspondances et à l'ensemble des hybridations de ces formes au sein de l'espace autobiographique.

D'une part, l'étude génétique permet de mesurer quelles opérations ont conduit à l'élaboration de l'œuvre; d'autre part, elle offre un «terrain de vérification privilégié aux engagements pris avec le pacte autobiographique ${ }^{1} \gg$. Elle implique donc un vaste terrain d'observation, qui prend d'abord en compte l'apport de sources externes : comme le note Lejeune, «le travail de la citation, les réemplois; les allusions, parfois même les plagiats [...] fourniront matière à travail génétique ${ }^{2} »$. Car tout autant que la littérature romanesque - et malgré les déclarations de singularité de Rousseau -, les écritures de soi sont un terrain poreux à l'intertextualité : plusieurs ouvrages autobiographiques usent d'ailleurs de citations ou d'intertextes parodiques en guise de titre, tels Je me retournerai souvent... de Dominique Arban (1990) ou encore les Mémoires d'une jeune fille dérangée de Bianca Lamblin (1993).

L'intertexte est la partie immergée de l'iceberg; l'ouvrage collectif Le Moi et ses modèles, sous-titré Genèse et transtextualités (2009), s'attachait à travers des études de cas menées sur Barthes, Gide, Queneau, Perec, Pozzi et Roubaud à cerner ces mécanismes : «Les avant-textes portent témoignage des sources, des modes d'appropriation, au fil du processus de création; ils reflètent des lectures, des influences, des esthétiques dont l'œuvre finale est susceptible ou non de garder trace. Dis-moi qui tu lis, je te dirai qui tu es ${ }^{3}$ », notaient Catherine Viollet et Véronique Montémont. Nommer ses lectures, les citer, les mettre en scène est d'ailleurs devenu, de Rousseau à Sartre en passant par Camus et Sarraute, un véritable topos du récit de formation, et nombreux sont les auteurs qui avouent leur passion enfantine, leur fascination adolescente ou leur dette littéraire définitive envers tel ou tel écrivain dont la découverte a été pour eux un éblouissement.

Cependant, Hélène Jaccomard, dans Lecteur et lectrice dans l'autobiographie française, souligne que «si l'autobiographie littéraire est un véritable hymne à la lecture $4^{4}$ et qu' on y voit souvent un narrateur qui «se met en scène en tant que lecteur de ses livres favoris ${ }^{5}$ », c'est aussi

1. Philippe Lejeune, Autogenèses. Les brouillons de soi, 2, Paris, Seuil, coll. «Poétique», 2013, p. 16.

2. Ibid., p. 345 .

3. Véronique Montémont et Catherine Viollet, «Avant-propos», Le Moi et ses modèles. Genèse et transtextualités, Louvain-la-Neuve, Academia-Bruylant, coll. «Au coeur des textes», 2009, p. 6.

4. Hélène Jaccomard, Lecteur et lecture dans l'autobiographie française contemporaine, Genève, Droz, 1993, p. 347.

5. Ibid., p. 349. 
«au lecteur et non à l'écrivain de reconnaître qu'il y a intertexte ${ }^{6}$ ». On touche là une limite de la notion d'intertextualité, telle qu'elle est défendue, par exemple par Kristeva, Riffaterre ou Barthes ${ }^{7}$ : d'abord, elle renvoie à la question de «formes plus ou moins reconnaissables ${ }^{8}$, comme l'écrit Barthes dans l'article «Théorie du texte» de L'Encyclopoedia Universalis, donc à un effet de réception; plus subrepticement, elle tend à laisser dans l'ombre un matériau non littéraire qui relève aussi bien de l'intratexte que de l'intertexte, fait de brouillons et de notes, de documents privés, tels qu'archives familiales, correspondances, cartes postales, photographies, tracts, prospectus, par définition inconnus du lecteur, mais qui participent pleinement à la fabrication de l'œuvre. Ici, la notion d'une transtextualité telle que définie par Genette («tout ce qui met en relation, manifeste ou secrète, [le texte] avec d'autres textes ${ }^{9}$ ») semble plus opératoire pour rendre compte de la multiplicité de ce feuilletage textuel.

C'est précisément sur la question d'une relation manifeste ou secrète soulevée par Genette que la génétique peut prendre le relais de l'analyse intertextuelle avec profit dans les écritures de soi. Comme l'explique Pierre-Marc de Biasi, «l'exogenèse ne désigne pas les "sources" de l'œuvre (telle personne réelle, tel lieu, telle œuvre littéraire, etc.) mais la trace repérable de ces référents-sources en termes de documents (écrits ou transposés) qui se trouvent attestés dans le dossier de genèse 10 ». En conséquence, l'analyse exogénétique rendra compte de la présence de telle ou telle source dans une autobiographie, voire un journal, mais permettra surtout de comprendre comment l'auteur.e l'a travaillée, a incorporé d'autres voix, d'autres subjectivités.

Questionner les rapports entre exogénèse et écritures de soi peut se faire sous plusieurs angles. D'une part, on peut s'interroger sur la capacité des écritures de soi à servir ellesmêmes d'éléments exogénétiques, lorsqu'elles prennent place dans un roman ou une œuvre de fiction; d'autre part, on peut considérer les écritures de soi comme terrain d'études génétiques où identifier des opérations d'exogenèse, ce qui revient à poser la question : comment parler de soi avec les mots des autres?

\section{Les journaux et les correspondances comme éléments exogénétiques}

Les journaux et correspondances sont des éléments exogénétiques relativement classiques, convoqués en ce qu'ils sont des tiers-lieux, «à la fois hors du livre et hors de la vie 11 ». Ils vont fournir des matériaux que le romancier peut conserver à l'identique ou réécrire. On observe deux modalités d'utilisation possibles : dans le cas A, ils se convertissent en matière endogénétique et le lecteur de l'œuvre publiée ne peut les identifier comme tels,

\footnotetext{
6. Ibid., p. 346.

7. Julia Kristeva, Sèméiotikè. Recherches pour une sémanalyse, Paris, Seuil, coll. «Tel Quel», 1969; Michael Riffaterre, La Production du texte, Paris, Seuil, coll. «Poétique», 1979, et Sémiotique de la poésie [Semiotics of poetry, 1978], traduit de l'américain par Jean-Jacques Thomas, Paris, Seuil, coll. «Poétique», 1983.

8. Roland Barthes, «Théorie du texte», Encyclopadia Universalis, t. XV, 1973.

9. Gérard Genette, Palimpsestes. La littérature au second degré, Paris, Seuil, coll. «Point», 1982, p. 5.

10. Pierre-Marc de Biasi, «Qu'est-ce qu'un brouillon? Le cas Flaubert : essai de typologie fonctionnelle des documents de genèse», dans M. Contat et D. Ferrer (éd.), Pourquoi la critique génétique? Méthodes, théories, manuscrits, Paris, CNRS Éditions, coll. «Textes et manuscrits», 1998, p. 31-60 (en ligne sur cairn.info).

11. Norbert Dodille, «Images de soi : autobiographie et autoportrait au XIX`e siècle », Romantisme, nº 56, 1987, p. 39.
} 
à moins de bénéficier des éclairages d'une étude génétique; dans le cas B, ils maintiennent, même dans l'œuvre publiée, leur statut de greffon.

Cas A : dans La Porte étroite (1909), André Gide, lui-même diariste et épistolier, fait tenir un journal ou écrire des lettres à ses personnages, Jérôme et Alissa. Or, Gide transpose dans ce récit fictionnel les années de crise morale et spirituelle qui ont précédé son mariage avec Madeleine Rondeaux et dont leurs journaux et lettres respectifs ont gardé la mémoire. Pour écrire le récit, Gide a relu ses propres lettres, celles qu'il avait adressées à Madeleine, mais il ne les utilise pas directement. En revanche, le journal de jeune fille et les lettres de Madeleine Rondeaux apparaissent comme des pilotis du récit. Si le journal d'Alissa porte la mémoire de celui de Madeleine tout en étant largement transposé dans le sens d'une amplification mystique et tragique, les lettres d'Alissa à Jérôme empruntent littéralement des passages de lettres de Madeleine Rondeaux et fournissent un exemple spectaculaire d'allogreffe épistolaire dont le lecteur du simple texte publié ne peut cependant rien deviner.

Cas B : les éléments exogénétiques initiaux conservent dans le texte publié leur statut exhibé de greffon. C'est ce que l'on trouve par exemple dans le roman naturaliste conçu comme un roman documentaire. Comme le note Pierre-Jean Dufief, «les Goncourt assimilent le travail de romancier à celui de l'historien; pour eux les historiens sont les raconteurs du passé, les romanciers ceux du présent. De leur pratique de l'histoire, nos auteurs vont conserver le goût du document, de la lettre autographe ${ }^{12}$.» On observe dans Søur Philomèle l'utilisation par les Goncourt d'une correspondance fournie par leur domestique, dans La Fille Élisa celle d'un paquet de lettres adressées à des prostituées et trouvées lors de la démolition d'une maison close. Chez les Goncourt, la lettre ne se fond pas dans la narration.

Isolée par des formules codifiées, [elle] n'est pas intégrée au récit; elle fait l'objet d'un chapitre autonome, elle est souvent présentée sans aucune focalisation, sans aucune mise en perspective, sans aucun recours à des personnages de scripteur ou de lecteur. [...] La présence des lettres contribue donc à la déconstruction du roman, à son éclatement en courts chapitres, à la naissance d'une forme hybride qui juxtapose des fragments hétérogènes mais toujours savamment travaillés ${ }^{13}$.

Le résultat obtenu n'a rien à voir avec un roman épistolaire mais serait plutôt du côté du roman-collage.

Un tel processus d'exogenèse peut également se rencontrer dans des mémoires ou autobiographies. C'est le cas où des écritures de soi vont féconder d'autres écritures de soi et où, le plus souvent, les avant-textes des mémoires ou autobiographies d'un auteur $\mathrm{X}$ vont comporter des lettres et journaux d'un auteur $\mathrm{Y}$ ou - cas extrême qui nous fait basculer de l'intertextualité dans l'intratextualité - de ce même auteur X (il en va ainsi des Mémoires de Simone de Beauvoir qui font intervenir des citations de son journal).

Un exemple est offert par les deux premières parties, sur les cinq que comporte le récit, d'Histoire de ma vie (1854-1855) de George Sand. Celles-ci vont être consacrées à la «préhistoire » de George Sand, en l'occurrence l'histoire de ses parents, et plus précisément celle de sa grand-mère paternelle - Marie-Aurore Dupin de Francueil, fille naturelle du Maréchal de

12. Pierre-Jean Dufief, «La lettre dans les romans des Goncourt : de l'effet de réel à la fétichisation de l'autographe», L'Esprit créateur, hiver 2000, vol. XL, no 4, p. 62.

13. Ibid., p. 61 


\section{Exogenèse de textes autobiographiques et mémoriels publiés}

Saxe - et de son père, Maurice Dupin. Parmi les divers documents que Sand a intégrés à son récit, la correspondance échangée entre son père et sa grand-mère de 1794 à 1808 occupe une place prédominante. Aussi peut-on dire que dans Histoire de ma vie, l'archive épistolaire est non seulement à l'origine du projet autobiographique, de son idée et de son désir, mais qu'elle en est aussi un des matériaux de construction essentiels. L'archive familiale - même si elle a été beaucoup retravaillée par Sand - est là à titre de document qui donne au texte autobiographique sa dimension historique ${ }^{14}$.

Comme l'a montré Philippe Lejeune ${ }^{15}$, bien que la proclamation de singularité absolue faite par Rousseau étende son ombre portée sur les écritures de soi depuis la fin du XVIIIe siècle, il n'en reste pas moins que le texte autobiographique écrit ex nihilo est un mythe : comme tout livre, il est hanté par la mémoire des textes lus, avec laquelle ou contre laquelle on écrit. Derrière les œuvres autobiographiques ou mémorielles, il y a une bibliothèque, voire des carnets ou des notes de lecture, que l'auteur convoque plus ou moins consciemment. Dans «L'autobiocopie», Philippe Lejeune étudie les exemples rares où l'auteur met lucidement en scène le fait que son autobiographie n'est qu'un tissu de réemplois de textes d'autrui : il en va ainsi de Marcel Bénabou dans Pourquoi je n'ai écrit aucun de mes livres (1986). Si ce cas extrême, presque parodique, spectacularise l'intertextualité, il n'a pas empêché que l'autobiographe extra-lucide soit lui-même pris à son propre jeu : l'auteur reconnaît dans un entretien avec Philippe Lejeune que les appropriations conscientes sont fondues «dans une prose elle-même faite de réemplois inconscients $16_{\gg \text {. }}$

Mais il existe nombre de cas où le texte autobiographique n'exhibe pas le processus exogénétique ou n'en donne qu'un trompe-l'œil. Il pourrait être intéressant à ce titre de comparer la présence effective de l'intertextualité dans le texte publié et le processus exogénétique tel que l'on peut le reconstituer dans les brouillons et les manuscrits de travail. Une œuvre d'Annie Ernaux peut nous servir d'exemple : L'Autre Fille, texte publié en 2011 sous la forme d'une lettre adressée par l'auteure à sa sœur Ginette, une sœur morte avant qu'elle-même ne naisse et dont elle a appris par hasard l'existence à l'âge de dix ans, en écoutant à la dérobée une conversation de sa mère avec une voisine. On peut comparer les références intertextuelles visibles dans le texte définitif avec les enseignements de l'avanttexte. Dans le texte publié de L'Autre Fille 17 on relève la présence de Flannery O'Connor (dans l'épigraphe : «La malédiction des enfants, c'est qu'ils croient »), d'Autant en emporte le vent (à cause de la mort de la petite fille de Scarlett et de Rhett [AF, 13]), du Journal de Pavese (AF, 19) de la collection Brigitte (AF, 33) de Claudel («Oui, je crois que je ne suis pas venue au monde pour rien et qu'il y avait en moi quelque chose dont le monde ne pouvait

14. Voir Brigitte Diaz, «Archives familiales et autobiographie dans Histoire de ma vie de George Sand» dans $\mathrm{V}$. Montémont et C. Viollet (dir.), Archives familiales : modes d'emploi. Récits de genèse, Paris, AcademiaL'Harmattan, 2013, p. 11-23.

15. Voir Philippe Lejeune, Les Brouillons de soi, op. cit., et «Écriture de soi et lecture de l'autre» dans J. Poirier (dir.), Écriture de soi et lecture de l'autre, Éditions universitaires de Dijon, 2002, p. 213-221.

16. Philippe Lejeune, «Autobiocopie», dans Les Brouillons de soi, op. cit., p. 25.

17. Annie Ernaux, L'Autre Fille, Paris, Nil, 2011. Toutes les références ultérieures à ce texte seront données ici entre parenthèses après la citation. 
se passer» AF, 35), de Bossuet («tu n'as été envoyée sur terre, comme l'écrit Bossuet, que "pour faire nombre" », AF, 37), de Kafka («Lettre au père », AF, 47), de l'évangile de Matthieu (AF, 56), de la «Consolation à M. Du Périer» de Malherbe (AF, 56), de Jane Eyre à cause de la mort d'Helen Burns, l'amie de jeunesse de Jane (AF, 64), de Peter Pan (AF, 76) sans oublier un dessin de Reiser : «Le pont des enfants perdus » (AF, 39). On note les multiples formes de cette intertextualité, qui mêle des références classiques, d'autres célèbres, d'autres appartenant à la littérature jeunesse (ou considérée comme telle) et une référence à une littérature populaire très prisée dans les années 1950 (la collection «Brigitte»). Le paysage intertextuel de l'avant-texte est sensiblement différent, à la fois plus dense et encore plus hétérogène : à certaines références présentes dans le texte publié (Peter Pan, Jane Eyre, Pavese, Bossuet, entre autres) s'ajoute la présence de la chanson et surtout de ce qu'on pourrait appeler «la chanson adressée » : Maxime Le Forestier et «Mon frère», Claude Nougaro et «Chanson pour Marylin », en accord avec la forme épistolaire choisie pour L'Autre Fille. Sont également mentionnés Mallarmé, Freud, Canetti, Virginia Woolf, Sartre, autant de grandes figures cautionnées par la culture «dominante» et dont le texte publié in fine ne portera pas trace.

Quels sont les rôles de ces pilotis invisibles de l'œuvre future? Ils peuvent fournir des exemples de mises en scène énonciatives (les chansons adressées). Mais il s'agit aussi d'élire certaines formules comme constat et mise en garde : elles deviennent quasiment des notes de régie de l'écrivain. Telle est sans doute la fonction prêtée à une phrase de Virginia Woolf, dans La Promenade au phare, qui apparaît plusieurs fois dans l'avant-texte : «les morts sont à notre merci ». Au verso de la chemise qui enserre le manuscrit 18 daté de 2010 , on lit une citation deFreud qui remplit un rôle constatif analogue : «Aucun événement n'est vécu en pure perte. Rien. Sauf la mort. » Sans doute certaines de ces citations constituent-elles également des rencontres inspirantes : on trouve chez un auteur la formule qui exprime ce que l'on n'avait pas encore verbalisé dans le miroir du langage, ce qui va mettre en branle l'écriture. Cela pourrait être le cas de ces phases de Canetti recopiées par Ernaux sur une demi-page A419 : «L'instant de survivre est instant de puissance. L'effroi d'avoir vu la mort se dénoue en satisfaction, puisque l'on n'est plus soi-même le mort [...] Masse et puissance.» Autant de citations qui sont l'amorce d'un dialogue avec d'autres voix et qui vont remplir pour Annie Ernaux une fonction heuristique. C'est donc sur un arrière-plan de thanatographie empruntée à divers penseurs qu'Annie Ernaux va pouvoir faire advenir le dévoilement d'un réel vertigineux, l'existence d'une sœur aînée morte avant sa propre naissance, d'une éternelle petite fille.

Lorsque le texte autobiographique choisit de donner à voir le processus d'intégration de ses sources, laissant subsister comme tel dans le texte publié le greffon, et que ce dernier se présente comme un texte factuel ou un document, il y a sans doute de la part de l'auteur la volonté d'afficher l'espace d'écriture dans lequel il se situe : la non-fiction. Mais cette problématique est néanmoins plus complexe qu'une simple dynamique de (re)production de la preuve : dans la procédure de transformation qui mène inévitablement du document manuscrit

18. «Manuscrit 2010», Bibliothèque nationale de France, NAF 28647.

19. La demi-page figure dans la chemise qui enserre le «Manuscrit $2010 »$.

\section{Transformations et enjeux}


au texte imprimé, il existe plusieurs degrés, et l'étude génétique est parfois nécessaire pour en éclairer les enjeux.

La démarche exogénétique présente dans le cas des écrits personnels une spécificité. Elle s'appuie en effet fréquemment sur des documents privés, la plupart du temps inaccessibles au lecteur, ce qui annule toute possibilité de (re)connaissance des sources de la part du public, aussi expert et qualifié soit-il. Si le narrateur opte pour le cas «A» évoqué plus haut, et qu'il faut se référer aux états manuscrits de l'élaboration du récit pour mesurer l'apport documentaire, le récit autobiographique court le risque de ne pas exploiter au mieux le capital de crédibilité que lui conférerait l'utilisation d'éléments exogènes, voire de laisser affleurer le soupçon d'une réappropriation effectuée de manière plus ou moins biaisée. Il existe d'ailleurs des litiges récurrents autour de l'utilisation silencieuse des écrits personnels d'autrui à titre de matériau génétique : le romancier Éric Reinhardt a été publiquement mis en cause en mars 2016 par l'avocate du modèle de son roman L'Amour et les forêts (2015), après qu'il eut repris de manière reconnaissable certains éléments d'un texte privé de quarante-quatre pages confié par cette correspondante.

L'étude génétique des manuscrits de Christine Angot déposés à l'IMEC, telle qu'elle a été exposée par Willian Vieira au séminaire «Autobiographie et correspondances », est un lieu où la sensibilité éthique et juridique de ces problématiques se révèle dans toute sa complexité, en particulier chez une auteure qui mêle sans cesse le document réel et sa refabrication littéraire. W. Vieira a en effet pu retracer l'existence d'un processus, qui consiste, de la part d'Angot, à reprendre, voire à susciter la fabrication de sources exogènes (par exemple des lettres ou un entretien avec son ex-mari Claude, pour Sujet Angot). Dans L'Inceste (1999) l'écrivain se réapproprie le rapport de lecture de l'avocate de l'éditeur Stock. Mais elle entrecoupe le long passage cité d'adresses diverses aux protagonistes mentionnés, passant sans cesse du il au $t u$, ce qui brouille les frontières énonciatives de l'original. Un document de travail externe, produit après coup par un avocat, mais évidemment prépondérant dans le processus éditorial, est ici récupéré par le texte qui le subvertit. En effet, alors que l'avocate insiste sur le caractère litigieux de la totalité du livre et le danger qu'il y a à le publier, le lecteur qui tient L'Inceste entre ses mains ne peut que constater que l'éditeur a passé outre. Insérer le rapport dans le discours à la première personne, telle une visible provocation, permet d'exhiber le mécanisme de censure juridique qui a failli s'appliquer au livre : une posture polémique, et même une bravade, qui accrédite l'idée d'un désir de vérité empêché chez un auteur prêt à en découdre :

Je n'ai pas le droit de mettre les vrais noms, l'avocate me l'a interdit, ni les vraies initiales. «Ce manuscrit présente de manière récurrente, un problème lié à la divulgation de la vie privée des proches de l'auteur, notamment celle de sa fille Léonore, mineure, de son ex-conjoint, Claude, de son père [qui a entretenu avec elle - voir les longues descriptions en fin d'ouvrage - des rapports incestueux]. D'autres personnes voient également l'intimité de leur vie privée étalée au grand jour, avec force détails, notamment Marie-Christine Adrey, l'amante de l'auteur et "personnage principal" de l'ouvrage, la comédienne Nadine Casta, etc. [...] Elle ne veut pas que je t'appelle X. Ni son vrai nom, ni ses initiales. [...] Ni X, ni MCA, ni Marie-Christine Adrey, ni Aime CA. [...] Ta cousine. NC, Nadine Casta, haine c'est, ce cinéma, ce théâtre, ce fric-là. [...]»20. 
L'étude des manuscrits nuance cependant cette déclaration de résistance, en montrant qu'une partie des préconisations de l'avocate, comme la modification des noms propres, est globalement respectée dans les différentes œuvres de Christine Angot.

Même dans les cas les plus extrêmes, quand une archive, loin d'être un simple greffon, est transsubstantiée dans un livre dont elle forme l'intégralité du cœur, du corps et de la matière, l'enjeu de l'étude génétique consiste à comprendre comment se sont opérées son assimilation et son organisation. Marie Billetdoux, dans C'est encore moi qui vous écris (2009), a procédé à une sélection et une compilation de quarante années de correspondances, actives et passives, issues de destinataires multiples. L'écrivain a dévoilé à l'occasion d'une journée d'études comment elle avait collecté et regroupé ce matériau composite : il comprend en effet sa correspondance personnelle, des lettres de lecteurs, ses lettres d'enfant, conservées par sa mère, des coupures de presse, et même une photo d'échographie. Marie Billetdoux a relu, trié, assemblé, puis dicté le tout à une secrétaire, une opération d' «écriture orale» qui lui a permis de transformer le matériau brut en manuscrit de près $\mathrm{d}^{\prime}$ ' un mètre de haut 21 » : «Tout en dictant, j'entendais où ça n'allait pas, où ça ne se raccordait plus, où ça perdait de l'intérêt, j'avais développé une espèce d'acuité, d'hypervigilance, d'ubiquité. [...] je savais, dans l'instant, si ça se rangeait dans la grande Histoire ou si c'était du déchet ${ }^{22}$.» Sa démarche est autant celle d'une archiviste que d'un écrivain : à travers cet assemblage mosaïque, celui de la voix de l'auteur et des voix tierces, à travers cette façon de mettre les lettres en miroir les unes des autres, d'organiser leur suture et leur jointoiement, on voit se dessiner l'ensemble d'une vie d'enfant, de fille, de femme, d'amante, de mère et d'écrivain, qui apparente le texte, en définitive, à un autoportrait polyphonique.

De la même manière que Philippe Lejeune a pu parler d'espace autobiographique, on pourrait donc aujourd'hui parler d'espace métagénétique, parfois créé par les critiques et les éditeurs, parfois organisé par les écrivains eux-mêmes. Ce geste peut obéir à des nécessités diverses : explorer les arcanes de sa propre écriture, sur le plan intratextuel - ce que fait Annie Ernaux dans L'Atelier noir ${ }^{23}$-, ou permettre au lecteur de prolonger le dialogue avec l'œuvre, précisément dans une optique exogénétique. À partir du $\mathrm{xx}^{\mathrm{e}}$ siècle, la question de l'exogenèse dans les textes autobiographiques semble devenir de plus en plus centrale. Comme on vient de le voir, de l'archive implicite, dont seule la lecture des manuscrits de l'œuvre ou des sources privées permettrait de retracer la part, on glisse d'abord vers une présence croissante, et surtout toujours plus visible du document personnel ou intime au sein du récit de soi. L'écrivain affiche un procédé d'exogenèse sur lequel il était auparavant plus discret. Souvent, loin de chercher à effacer l'échafaudage documentaire, l'auteur prend soin d'en dévoiler la mise en place, nourrissant parfois un récit secondaire, celui de l'intégration des traces au processus d'écriture. Le travail de Michel Foucault, avec la publication de Moi, Pierre Rivière (1973),

\section{L'exposition de l'archive : l'espace métagénétique}

21. Marie Billetdoux, «Le treillis de lumière», «Archives familiales et autobiographie dans Histoire de ma vie de George Sand» dans V. Montémont et C. Viollet (dir.), Archives familiales : modes d'emploi. Récits de genèse, Paris, Academia-L'Harmattan, 2013, p. 50.

22. Ibid., p. 50.

23. Il s'agit de son journal d'écriture : Annie Ernaux, L'Atelier noir, Aurillac, éditions des Busclats, 2011. 
d'Arlette Farge, avec son Goût de l'archive (1997) ou encore de Michèle Perrot, dont on peut citer Mélancolie ouvrière (2012), a suscité un regain d'intérêt pour des écritures dites «ordinaires » (qui dans les faits ne le sont pas toujours) et ont contribué à extraire le document du statut ancillaire qui lui était jusque-là souvent réservé. Par contrecoup, ce dernier a gagné en légitimité; non seulement il ne délittérarise pas l'œuvre dans laquelle on l'insère, mais de plus, en contexte autobiographique, il vient renforcer le versant référentiel du pacte.

Le livre emblématique de cette démarche est naturellement le Roland Barthes par Roland Barthes (1975), avec son album photo liminaire, mais aussi ses lettres, dessins, caricatures et extraits manuscrits, qui ouvre la voie à un usage de plus en plus réfléchi de l'archive dans le processus de création. Anne Herschberg Pierrot a d'ailleurs montré comment l'étude des brouillons du Rolland Barthes par Rolland Barthes fait apparaître une écriture de type diaristique, avec des dates, des entrées, des réflexions sur le travail en cours, supprimées de la version finale 24 . On retrouve une semblable logique chez Philippe Artières, qui prend soin dans Vie et Mort de Paul Gény de faire figurer la reproduction de la correspondance de son grand-oncle, de recopier la liste des intitulés de plaques commémoratives auxquelles il a pensé, de reproduire les lettres et le cahier de l'assassin de Paul Gény, Bambino Marchi, avec les photos que comprenait le document original. La description liminaire du document, soigneuse, ne déparerait pas les premières lignes d'un article scientifique, car elle est aussi un exposé génétique minutieux :

Un grand cahier broché, semblable à ces registres d'institution, dont chaque page est numérotée.

Celui-là est de format 21 x 39,7 cm et compte 96 pages de petits carreaux sans marge imprimée.

Sur la couverture toilée de couleur marron avec une reliure noire, aucune autre indication ne figure que le tampon de l'hôpital San Lazzaro. Dans le cahier, seule la moitié des pages est utilisée25.

Cette fois, la trace n'est plus seulement dans les avant-textes cachés : fac-similés, dessins, photographies, lettres, notes manuscrites abondent et sont mis en avant. Cette présence, cette démarche réflexive sont sans aucun doute à mettre en rapport avec l'appropriation de plus en plus fréquente du genre autobiographique par les historiens. Après l'ego-histoire, qui les invitait à s'exprimer sur leur biographie, une hétérohistoire, entre biographie, autobiographie et récit de filiation, s'incarne sous la plume de Mona Ozouf (Composition française, 2008), Martine Sonnet (Atelier 62, 2008), Stéphane Audoin-Rouzeau (Quelle histoire. Un récit de filiation [1914-2014], 2013) ou Ivan Jablonka (Histoire des grands-parents que je n'ai pas eus, 2012) ${ }^{26}$. On pourrait dire que les auteurs proposent, en somme, dans le même espace, le texte et son commentaire, le récit final et son making of sous la forme d'une enquête. Le lecteur marche sur les pas de l'écrivain, partage sa ferveur et ses doutes, espérant comme lui que des documents jaillira la lumière :

\footnotetext{
24. Barthes a par exemple amputé le fragment «Le matin à U. » d'une notation datée à caractère réflexif : «Ce matin, l'ivresse même est accrue par l'optalidon que j'ai pris [...] et ce peu de corydrane que je me suis permis dans l'espoir de vaincre la corvée de relire tous mes livres, en vue d'écrire ce R.B par lui-même» (c'est le premier jour de cette tâche, 6 août 1973). Voir Anne Herschberg Pierrot, «Les manuscrits de Roland Barthes par Roland Barthes. Style et genèse », Genesis, no 19, «Roland Barthes», 2002, p. 191-215 (en ligne sur persee.fr).

25. Philippe Artières, Vie et Mort de Paul Gény, Paris, Seuil, 2013, p. 154.

26. Voir Véronique Montémont, «Historien de soi », Revue des sciences humaines, no 334, «Les Formes de l'enquête», D. Méaux (dir.), 2019, Villeneuve-d'Ascq, Presses universitaires du Septentrion, p. 147-157.
} 
Les dossiers suivants sont aussi pleins de surprises, ils ne contiennent pas moins de trois expertises mentales de quelque six médecins dont la dernière fait près de deux cents pages dactylographiées. J'entreprends de photographier l'ensemble; je m' applique pour ne pas faire des clichés flous, inutilisables... évidemment ma batterie se vide et je me retrouve dans l'impossibilité de finir la production ${ }^{27}$.

Armé de mon ordinateur et de mon appareil photo numérique, je saute de bon matin dans un train à la gare de Lyon et me voilà parti à l'assaut du fichier de la Sûreté nationale28.

Les matériaux exogénétiques ont quitté les coulisses et sont volontiers spectacularisés.

Intertexte et exogenèse sont deux mécanismes très présents dans les écritures de soi, avec des enjeux différents de ceux présents dans la fiction. Des matériaux composites, qui empruntent aussi bien à l'intertexte qu'à l'intratexte - notamment celui des différents supports d'écriture privés de l'auteur, tels le journal, la correspondance ou les notes de travail -, forment autant d'embrayeurs d'écriture qui pourront être transformés, assimilés, se fondant dans le texte d'arrivée une fois qu'ils y auront joué leur rôle. L'examen génétique montre comment s'est opérée cette intégration, qui ne va pas sans questionnements ni remaniements parfois profonds du texte original. Toutefois, on peut constater que la présence de traces documentaires a majoritairement évolué vers le deuxième cas envisagé au début de cet article, celui du greffon qui a conservé son statut, et qui souvent le revendique.

L'autobiographie contemporaine, en accentuant l'exposition de ces mécanismes exogénétiques, en les transformant parfois en matrice d'un récit secondaire, a donc dégagé en son propre sein une étape discursive où est régulièrement questionnée la construction de la mémoire. Bien sûr, il faut se garder d'une forme de naïveté qui consisterait à croire qu'évoquer un document, le donner à lire ou même le reproduire dans l'espace du livre, offre une garantie unilatérale de vérité : ces traces sont sujettes à interprétation, parfois même à caution, et il n'est pas interdit, comme le montrent les exemples d'Angot, de les détourner ou d'en fabriquer! Cependant, il semblerait que la seconde moitié du xxe siècle, et plus encore le $\mathrm{XXI}^{\mathrm{e}}$, ait privilégié cette esthétique de la métagenèse. Parmi les raisons qui l'expliquent, on peut citer la succession des guerres mondiales, génocides et diasporas, qui ont déplacé des populations entières, rendant l'oubli intolérable et les traces familiales infiniment précieuses. Mais des évolutions techniques, entre la composition par ordinateur et la possibilité de publier des sources intermédiales sur le web, ont également joué leur rôle. Elles ont autorisé une maniabilité plus grande du document, qu'il est aisé et peu onéreux de mettre à disposition.

Surtout, l'autobiographie, à partir des années 1970, a connu un fort virage réflexif, que l'émergence de l'autofiction a rendu plus central encore. Marc Angenot écrivait en 1983 :

L'approche «intertextuelle» peut avoir pour effet de briser la clôture de la production littéraire canonique pour inscrire celle-ci dans un vaste réseau de transactions entre modes et statuts discursifs, le discours social. Il y a là une attitude nouvelle quant à la place même qu'occupe le littéraire dans l'activité symbolique 29 .

27. Philippe Artières, op. cit., p. 117.

28. Ivan Jablonka, Histoire des grands-parents que je n'ai pas eus, Paris, Seuil, 2012, p. 132.

29. Marc Angenot, «L'intertextualité : enquête sur l'émergence et la diffusion d'un champ notionnel», Revue des sciences humaines, $\mathrm{n}^{\circ} 89,1983$, p. 128. 
L'autobiographie, envisagée sous cet angle, peut bénéficier de nouveaux questionnements : sa relation au matériau intertextuel interroge ses limites génériques dans la sphère des écrits personnels, mais tend aussi à renégocier une relation au réel qu'on avait pu croire affaiblie par l'émergence de l'autofiction. L'exogenèse, parce qu'elle met en évidence la relation aux textes tiers qui nourrissent l'autobiographie, et surtout l'ensemble de la réflexion qui entoure leur appropriation, fait au contraire souvent apparaitre une solidarité profonde du réel et de ses traces, une volonté mémorielle aiguë, un texte conçu, de plus en plus souvent, comme un lieu de parole polyphonique, qui assume la pluralité de ses énonciateurs et même s'en enorgueillit. S'écrire en questionnant les procédures d'élaboration de son discours, placer le lecteur au cœur de l'enquête et de l'écriture : voici aujourd'hui, à l'ère du soupçon autofictionnel, une autre façon de signer le pacte.

Françoise Simonet-Tenant enseigne la littérature française du xx siècle à l'Université de Rouen. Elle est l'auteur d'études critiques : Le Journal intime (2001) et Journal personnel et correspondance (1785-1939) ou les affinités électives (2009), et a dirigé plusieurs ouvrages collectifs ou numéros de revue : Le propre de l'écriture de soi, éditions Téraèdre, 2007; Genesis, ${ }^{\circ} 32$, « Journaux personnels », SUP, mars 2011; Annie Ernaux : l'intertextualité, Mont-Saint-Aignan, Presses universitaires de Rouen et du Havre, 2015 ; «Ce qui parle en moi » : l'étrangeté de la voix, Revue des Sciences Humaines, $\mathrm{n}^{\circ} 333,2019$; Dictionnaire de l'autobiographie. Écritures de soi de langue française, paru chez Champion en mai 2017.

francoise.simonet-tenant6@orange.fr

VÉRONIQUE MONTÉMONT est maître de conférences HDR en langue et littérature françaises à l'Université de Lorraine. Elle coanime avec Jean-Marc Hovasse l'équipe «Autobiographie et correspondances» (ITEM) depuis 2015. Elle a également créé et anime depuis 2016 le blog Autobiosphère (https://autobiosphere.wordpress.com). Elle a codirigé plusieurs ouvrages collectifs sur l'autobiographie et le journal personnel et a participé à la rédaction du Dictionnaire de l'autobiographie française et francophone (dir. F. Simonet-Tenant, Champion, 2017).

veronique.montemont@orange.fr 


\section{Résumés \\ L'espace métagénétique : exogenèse, intertextualité, écritures de soi}

Les écritures de soi sont des objets pour lesquels l'étude génétique peut prendre avec profit le relais de l'analyse intertextuelle, en ce que cette dernière repose essentiellement sur la capacité que le lecteur a d'identifier les discours tiers. Or, dans le cas des écrits personnels, les discours tiers sont loin d'être toujours repérables, parfois privés, parfois inédits. L'analyse exogénétique, elle, rendra compte de la présence de telle ou telle source dans une autobiographie, voire un journal, mais permettra surtout de comprendre comment l'auteur.e l'a travaillée, a incorporé d'autres voix, d'autres subjectivités. Cet article envisagera donc la question sous une double perspective : examiner, d'un côté, comment les écritures de soi, journaux ou correspondances, peuvent prendre place dans une œuvre de fiction; analyser, de l'autre, la façon dont l'écriture de soi met en scène, de plus en plus explicitement, les traces et matériaux extérieurs dont elle se nourrit, produisant ainsi un véritable récit métagénétique. Ce qui revient à poser la question : comment parler de soi avec les mots des autres?

Applying genetic analysis to self-writing can profitably replace and extend intertextual analysis, insomuch as the latter relies upon the ability of the reader to identify exogenous discourses. In the case of self-writing, these are far from being self-evident; at times, they come from a personal archive, and, at times, they are based on unpublished material. Exogenetic analysis locates the presence of a variety of sources in an autobiography, such as a diary that can inform the published text. But it also reveals how the author refashions these sources, incorporating other voices and other subjectivities. This article has two goals: first, to examine how personal writing, such as diaries and correspondence, can be incorporated into fiction; second, to analyze how personal writing increasingly shows the traces and external materials that sustain it and that lead to a genuinely metagenetic narrative. The question returns: How is it that the words of others allow us to talk about ourselves?

Persönliche Schriften sind Objekte, bei denen die genetische Untersuchung die intertextuelle Analyse gewinnbringend ablösen kann, da letztere im Wesentlichen auf der Fähigkeit des Lesers beruht, die Diskurse Dritter zu identifizieren. Im Falle von persönlichen Schriften sind die Diskurse Dritter jedoch bei weitem nicht immer erkennbar, manchmal privat, manchmal unveröffentlicht. Die exogenetische Analyse hingegen wird über das Vorhandensein dieser und jener Quelle in einer Autobiographie oder sogar in einem Tagebuch Rechenschaft ablegen, vor allem aber wird sie es ermöglichen, zu verstehen, wie der Autor sie bearbeitet, wie er andere Stimmen, andere Subjektivitäten einbezogen hat. In diesem Artikel soll daher die Frage aus einer doppelten Perspektive betrachtet werden: Zum einen soll untersucht werden, wie die persönliche Schrift, sei es in Form von Zeitschriften oder Korrespondenz, seinen Platz in einem Werk der Fiktion einnehmen kann; zum anderen soll die Art und Weise analysiert werden, in der das Selbstschreiben die äußeren Spuren und Materialien, von denen es sich speist, immer expliziter inszeniert und so eine echte metagenische Erzählung hervorbringt. Das läuft darauf hinaus, die Frage zu stellen: Wie können wir mit den Worten anderer über uns selbst sprechen?
Las escrituras de sí mismo son objetos para los cuales el estudio genético puede sustituir ventajosamente al análisis intertextual, dado que este último se basa esencialmente en la capacidad del lector para identificar los discursos de terceros. Ahora bien, en el caso de los escritos personales, los discursos de terceros están lejos de ser siempre identificables - pueden ser privados, o bien inéditos. El análisis exogenético, por su parte, dará cuenta de la presencia de tal o tal fuente en una autobiografía - e incluso en un diario - pero sobre todo permitirá entender como el/la autor (a) la ha modificado, incorporando otras voces, otras subjetividades. Este artículo abordará entonces la problemática con una doble perspectiva: por un lado, considerar como las escrituras de sí mismo, diarios o correspondencias, pueden encontrar un lugar en una obra de ficción; por el otro, analizar la manera con que la escritura de sí mismo escenifica, de manera cada vez más explícita, las huellas y materiales exteriores de los que se alimenta, produciendo de este modo un verdadero relato metagenético; lo que equivale a plantear el interrogante: ¿cómo hablar de sí mismo con las palabras de otros?

No que diz respeito aos escritos pessoais o estudo genético pode substituir vantajosamente a análise intertextual, na medida em que esta última se baseia essencialmente na capacidade do leitor de identificar os discursos de terceiros. No entanto, no caso dos escritos pessoais, esses discursos de terceiros estão longe de ser sempre identificáveis, por vezes privados, por vezes inéditos. A análise exogenética dará conta da presença de determinada fonte numa autobiografia, ou mesmo num diário, mas permitirá sobretudo compreender como o autor a trabalhou, incorporou outras vozes, outras subjetividades. Este artigo considerará portanto a questão de uma dupla perspetiva: examinará, por um lado, como os escritos pessoais, diários ou correspondência, podem ter lugar numa obra de ficção; analisará, por outro lado, o modo como a escrita pessoal evidencia, cada vez mais explicitamente, vestígios e materiais externos de que se alimenta, produzindo assim uma verdadeira narrativa metagenética. Pelo que é pertinente perguntar: como podemos falar de nós próprios com as palavras dos outros?

Al posto dell' analisi intertestuale, che riposa essenzialmente sulla capacità del lettore d'identificare i discorsi esterni, le "scritture del sé" possono essere sottomesse con profitto all'analisi genetica. Nel caso degli scritti personali, infatti, i discorsi esterni, a volte privati o inediti, sono difficilmente rintracciabili. L'analisi esogenetica, dal canto suo, renderà conto della presenza di tale o talaltra fonte in un' autobiografia o un diario, ma soprattutto permetterà di capire come l'autore l'ha trattata, come ha incorporato altre voci, altre soggettività. L'articolo affronterà la questione da una doppia prospettiva: esaminerà, da un lato, come le scritture del sé (diari, lettere) possono inserirsi in un'opera di finzione; dall'altro, analizzerà il modo in cui la scrittura del sé mette in scena, sempre più esplicitamente, le tracce e i materiali esterni di cui si nutre, producendo così un vero e proprio racconto metagenetico. La questione si potrebbe riassumere con la domanda: come parlare di sé con le altrui parole? 